\title{
A NOTE ON THE LARGE DEVIATIONS FOR PIECEWISE EXPANDING MULTIDIMENSIONAL MAPS
}

\author{
R. AIMINO AND S. VAIENTI
}

\begin{abstract}
We provide the large deviation principle for higher dimensional piecewise expanding maps and by using the functional approach of Hennion and Hervé, slightly modified.
\end{abstract}

\section{INTRODUCTION}

There are different ways to establish large deviation principles (LDP) for dynamical systems. One of them is the so-called "Laplace Method", which relies on the spectral properties of the Perron-Frobenius, or transfer, operator. This strategy has been developed in a very general and abstract setting by Hennion and Hervé in [15]. They assume that the transfer operator acts on a Banach spaces of measurable functions, and it is quasi-compact on it, i.e. it has a spectral gap. The existence of an invariant probability measure follows immediately, and, by using perturbation theory for linear operators, they derive a few others statistical properties. This approach covers a lot of systems, for instance expanding maps of the interval [15, XII.1], Gibbs measures for subshift of finite type [15, XII.2], and expanding Young towers 29. Nevertheless, this theory seems inappropriate for expanding (discontinuous) maps in higher dimension, like those treated by several authors [1, 3, 5, 7, 8, 9, 21, 23, 25, 26, 27, 28]. Indeed, Hennion and Hervé assume that the Banach space on which the transfer operator acts, consists of measurable functions defined everywhere, but for the higher dimensional systems quoted above, the functional spaces usually considered (bounded variation, quasi-Hölder or Sobolev spaces) consist of classes of equivalence of functions modulo the reference measure, and hence they are only defined almost everywhere. Furthermore, in [15], the Dirac masses must belong to the topological dual of the Banach space, so this theory cannot be applied directly for those systems.

Nevertheless it appears that one could slightly modify the proofs from [15] in order to deal with a Banach space consisting of classes of functions. In particular we will

SV warmly thanks E. Ugalde for the kind invitation to participate to the Conference in honor of Valentin Afraimovich; RA and SV express their sincere gratitude to I. Melbourne who helped them to simply the proofs. 
do it for the functional space of quasi-Hölder functions mostly investigated in [25] and which verify an additional algebraic assumption which also plays a role in the Hennion-Hervé approach. As a consequence we will get the large deviations principle for such systems and, as far as we know, this result is not present in the literature. We also prove the central limit theorem, but for the latter one already disposes of the Gordin-Liverani theorems [13, 20].

Actually a weaker result for the large deviations of systems like those considered above has been recently obtained in [2]. We will comment about the difference with the spectral technique presented in this note in the Remark 2 below. We anticipate here that the paper [2] furnishes an upper bound for the deviation functions and whenever the correlation functions involving $L^{1}$ observables decay to zero with a summable rate. In order to check these assumptions for our systems we should further require that the density of the invariant measure is essentially bounded from below, but this assumption is not necessary in the spectral approach discusses later on.

\section{Assumptions And statement of the RESults}

We now give the precise assumptions under which the LDP is valid. Let $(X, \mathcal{A}, m)$ a probability space, and $T: X \rightarrow X$ a measurable transformation, non singular with respect to $m$. Under these conditions, the Perron-Frobenius operator $P: L^{1}(m) \rightarrow$ $L^{1}(m)$ is well defined by $P f=\frac{d m_{f}}{d m}$, where $m_{f}(A)=\int_{T^{-1} A} f d m$ is absolutely continuous with respect to $m$. We stress here the fact that the functions under consideration are complex valued, as required by the spectral theory we are going to use below. The transfer operator enjoys some classical properties that we resume below; see [4] or [18] for more details.

(1) Linearity: $P$ is a linear operator on $L^{1}(m)$, satisfying $\|P f\|_{1} \leq\|f\|_{1}$ for all $f \in L^{1}(m)$

(2) Positivity: For all $f \in L^{1}(m)$ such that $f \geq 0 m$-ae, we have $P f \geq 0 m$-ae;

(3) Preservation of integrals: For all $f \in L^{1}(m)$, we have $\int P f d m=\int f d m$;

(4) Duality: For all $f \in L^{1}(m)$ and $g \in L^{\infty}(m)$, we have $\int f(g \circ T) d m=$ $\int(P f) g d m$

(5) Invariant Measures : $f \in L^{1}(m)$ is the density of a $T$-invariant probability if and only if $f \geq 0, \int f d m=1$ and $P f=f$.

Let us suppose now that we have a subspace $\mathcal{B} \subset L^{1}(m)$, equipped with a norm $\|\cdot\|_{\mathcal{B}}$ such that

(1) $\left(\mathcal{B},\|.\|_{\mathcal{B}}\right)$ is a complex Banach space with continuous injection $\mathcal{B} \rightarrow L^{1}(m)$;

(2) Constant functions lie in $\mathcal{B}$; 
(3) $\mathcal{B}$ is a Banach algebra : there exists $C>0$ such that for all $f, g \in \mathcal{B}$ we have $f g \in \mathcal{B}$ with $\|f g\|_{\mathcal{B}} \leq C\|f\|_{\mathcal{B}}\|g\|_{\mathcal{B}}$

(4) $\mathcal{B}$ is a complex Banach lattice : for every $f \in \mathcal{B}$, we have $\bar{f},|f| \in \mathcal{B}$;

$(5) \mathcal{B}$ is stable under $P: P(\mathcal{B}) \subset \mathcal{B}$;

(6) $P$ is a bounded operator on $\mathcal{B}$, with spectral radius equal to one;

(7) $P$ is quasi-compact of diagonal type on $\mathcal{B}$.

The last assertion means that there exists a decomposition

$$
P=\sum_{i=1}^{s} \lambda_{i} \Pi_{i}+Q
$$

where $\lambda_{i}$ are complex numbers of modulus $1, \Pi_{i}$ are finite-rank projections satisfying $\Pi_{i} \Pi_{j}=0$ when $i \neq j$ and $Q$ is a bounded operator on $\mathcal{B}$ with spectral radius strictly less than 1 and satisfying $Q \Pi_{i}=\Pi_{i} Q=0$ for all $i$. The spectrum of $P$ consists then of a finite number of eigenvalues of modulus 1 , with finite multiplicity, and the rest of the spectrum lies in a disc centered at 0 with radius strictly less than 1 . When $\mathcal{B}$ is compactly injected in $L^{1}(m)$, this can be deduced from a Lasota-Yorke type inequality, by means of the Ionescu-Tulcea and Marinescu theorem [16, 14]. See [15] for precise definitions and results about quasi-compactness.

Under those conditions, the existence of an $T$-invariant probability $\mu$ absolutely continuous w.r.t $m$, such that $\frac{d \mu}{d m} \in \mathcal{B}$ is a classical result : for every $f \in \mathcal{B}$ such that $f \geq 0, \int f d m=1$, and so in particular for $f=\mathbb{1}$, quasi-compactness implies that the sequence $\frac{1}{n} \sum_{k=0}^{n-1} P^{k} f$ converges in $\mathcal{B}$ to a function $f^{\star}$ such that the measure $\mu_{f}$ with $\frac{d \mu_{f}}{d m}=f^{\star}$ is an acip. Furthermore, 1 is an eigenvalue of $P$. If we assume that 1 is a simple eigenvalue of $P$, then there exists an unique acip $\mu$ such that $\frac{d \mu}{d m} \in \mathcal{B}$. From now, we will always assume that 1 is a simple eigenvalue, and that there is no other eigenvalue of modulus 1 . $\mu$ will denote the unique acip, and $v \in \mathcal{B}$ its density. We then have 1 , for all $n \geq 1$ and $f \in \mathcal{B}$

$$
P^{n} f=<m, f>v+Q^{n} f
$$

As a consequence, we get exponential decay of correlation : there exists $C \geq 0$ and $0 \leq \lambda<1$ such that for every $f \in \mathcal{B}$ and every $g \in L^{\infty}(\mu)$ we have

$$
\left|\int f\left(g \circ T^{n}\right) d \mu-\int f d \mu \int g d \mu\right| \leq C \lambda^{n}\|f\|_{\mathcal{B}}|| g \|_{L_{\mu}^{\infty}}
$$

\footnotetext{
${ }^{1}$ When $\varphi \in \mathcal{B}^{\star}$ belongs to the topological dual of $\mathcal{B}$, we denote $\langle\varphi, f\rangle=\varphi(f)$. The linear form $f \rightarrow \int f d m$ belongs to $\mathcal{B}^{\star}$, and we denote it by $m$.
} 
Let now $\phi: X \rightarrow \mathbb{R}$ a bounded observable which lie in $\mathcal{B}$, with zero mean $\int \phi d \mu=$ 0. Denote by $S_{n}$ the Birkhoff sums :

$$
S_{n}=\sum_{k=0}^{n-1} \phi \circ T^{k}
$$

We are now able to state the LDP :

Theorem 1. (Large Deviation Principle)

Under the above conditions, the limit $\sigma^{2}=\lim _{n \rightarrow \infty} \int\left(\frac{S_{n}}{\sqrt{n}}\right)^{2} d \mu$ exists, and if $\sigma^{2}>0$, then there exists for some $\epsilon_{0}>0$ a rate function $\left.c:\right]-\epsilon_{0},+\epsilon_{0}[\rightarrow \mathbb{R}$, continuous, strictly convex, vanishing only at 0 , such that for every $0<\epsilon<\epsilon_{0}$ and every probability measure $\nu$ with $\nu \ll m$ and $\frac{d \nu}{d m} \in \mathcal{B}$, we have

$$
\lim _{n \rightarrow \infty} \frac{1}{n} \log \nu\left(S_{n}>n \epsilon\right)=-c(\epsilon)
$$

As an easy consequence of the techniques introduced in the next section, we also get the central limit theorem. We denote with $\mathcal{N}\left(0, \sigma^{2}\right)$ the Dirac mass $\delta_{0}$ if $\sigma^{2}=0$, and the probability with density $\frac{1}{\sigma \sqrt{2 \pi}} e^{-\frac{t^{2}}{2 \sigma^{2}}}$ with respect to Lebesgue if $\sigma^{2}>0$.

Theorem 2. (Central Limit Theorem) Under the same assumptions of Theorem $1, \frac{S_{n}}{\sqrt{n}}$ converges in distribution to $\mathcal{N}\left(0, \sigma^{2}\right)$ in the probability space $(X, \mathcal{A}, \nu)$ for every probability $\nu$ with $\nu \ll m$ and $\frac{d \nu}{d m} \in \mathcal{B}$ : for every bounded continuous function $g: \mathbb{R} \rightarrow \mathbb{R}$, we have

$$
\lim _{n \rightarrow \infty} \int g\left(\frac{S_{n}}{\sqrt{n}}\right) d \nu=\int g d \mathcal{N}\left(0, \sigma^{2}\right)
$$

\section{Remark 3.}

(1) Theorems 1 and 2 apply in particular for $\nu=m$ and $\nu=\mu$, so the LDP and the CLT are valid for both reference and invariant measures.

(2) As we anticipated in the Introduction, the paper [2] gives an upper bound for the large deviation function and under related assumptions. In particular Th. E in [2] states the following, with our notations. Let us suppose that $T$ preserves an ergodic probability measure $\mu$; then let $\mathcal{B} \subset L^{1}(\mu), \phi \in \mathcal{B}$, and assume that there exists $\xi(n)$ with $\sum_{n=0}^{\infty} \xi(n)<\infty$ such that for all $\psi \in$ $L^{1}(\mu)$ we have $\left|\int \phi\left(\psi \circ T^{n}\right) d \mu-\int \phi d \mu \int \psi d \mu\right| \leq \xi(n)\|f\|_{\mathcal{B}} \mid\left\|_{g}\right\|_{L_{\mu}^{\infty}}$. Then there exists $\tau=\tau(\phi)>0$ and, for every $\epsilon>0$, there exists $C=C(\phi, \epsilon)>0$ such that $\mu\left(S_{n}>n \epsilon\right) \leq C e^{-\tau n}$.

The proof of this result relies on a martingale approximation. Section $\mathrm{C}$ in [2] provides examples of systems for which one gets exponential decay of correlations (and hence $\xi(n)$ is summable) and against $\psi \in L^{1}(\mu)$ : this last 
assumption requires the density to be bounded from below. In conclusion the result of our note extends the previous one in two directions : first, we obtain a LDP, and not only an upper bound; secondly, we do not have to assume anything about the density, but the fact that it is in the $L^{1}$ norm with respect to the conformal (reference) measure.

\section{Proofs}

We begin by observing that the existence of the variance $\sigma^{2}$ results from a straightforward computation : since the sequence $\int \phi\left(\phi \circ T^{n}\right) d \mu$ decays exponentially fast, it is absolutely summable, and then we see, by expanding the term $S_{n}^{2}$, that the limit $\sigma^{2}=\lim _{n \rightarrow \infty} \int\left(\frac{S_{n}}{\sqrt{n}}\right)^{2} d \mu$ exists and we have

$$
\sigma^{2}=\int \phi^{2} d \mu+2 \sum_{n=1}^{+\infty} \int \phi\left(\phi \circ T^{n}\right) d \mu
$$

We assume from now $\sigma^{2}>0$. Our proof of the LDP follows closely [15] except for a minor modification, which will be mentioned later. The same approach had been employed in [24]. Let $f \in \mathcal{B}$ the density of the measure $\nu$ with respect to $m$. We will apply Gartner-Ellis theorem [10, 12], so we are interested in the convergence of the sequence $\frac{1}{n} \log \int e^{\theta S_{n}} f d m$ for $\theta \in \mathbb{R}$ small enough. We introduce the "Laplace transform" operators $P_{z}$, for $z \in \mathbb{C}$, defined by

$$
P_{z}(f)=P\left(e^{z \phi} f\right), f \in \mathcal{B}
$$

Assuming for a moment that $P_{z}$ is well defined, we see immediately that we have $\int e^{\theta S_{n}} f d m=\int P_{\theta}^{n}(f) d m$. In order to prove that $P_{z}$ is a bounded operator on $\mathcal{B}$, we just have to check that $e^{z \phi} \in \mathcal{B}$. Since $\mathcal{B}$ is a Banach algebra, the sequence $\sum_{k=0}^{n} \frac{(z \phi)^{k}}{k !}$ converges in $\mathcal{B}$, and hence in $L^{1}(m)$. On the other hand, this sequence converges uniformly, and hence in $L^{1}(m)$, to $e^{z \phi}$, and so we get that

$$
e^{z \phi}=\sum_{n=0}^{+\infty} \frac{(z \phi)^{n}}{n !}
$$

in $\mathcal{B}$. It also proves that the map $z \rightarrow P_{z}$ is holomorphic and we have the expansion

$$
P_{z}=\sum_{n=0}^{+\infty} \frac{C_{n}}{n !} z^{n}
$$

where $C_{n}(f)=P\left(\phi^{n} f\right)$.

We can now apply perturbation theory for linear operator to prove the following result. The proof relies on analytic functions of operators, see [11], or on the implicit function theorem, see [15]. For $\theta>0$, we denote $\mathbb{D}_{\theta}=\{z \in \mathbb{C} /|z|<\theta\}$. 
Proposition 4. There exist $\theta_{0}>0, C>0, \eta_{1}, \eta_{2}>0$ and holomorphic functions $\lambda():. \mathbb{D}_{\theta_{0}} \rightarrow \mathbb{C}, v():. \mathbb{D}_{\theta_{0}} \rightarrow \mathcal{B}, \varphi():. \mathbb{D}_{\theta_{0}} \rightarrow \mathcal{B}^{\star}$ and $Q():. \mathbb{D}_{\theta_{0}} \rightarrow \mathcal{L}(\mathcal{B})$ such that for all $z \in \mathbb{D}_{\theta_{0}}$

(i) $\lambda(0)=1, v(0)=v, \varphi(0)=m, Q(0)=Q$;

(ii) $P_{z}(f)=\lambda(z)<\varphi(z), f>v(z)+Q(z) f$ for all $f \in \mathcal{B}$;

(iii) $<\varphi(z), v(z)>=1$;

(iv) $Q(z) v(z)=0$ and $\varphi(z) Q(z)=0$;

(v) $|\lambda(z)|>1-\eta_{1}$

(vi) $\left\|Q(z)^{n}\right\| \leq C\left(1-\eta_{1}-\eta_{2}\right)^{n}$.

So, for all $n \geq 1$, we have

$$
P_{z}^{n}(f)=\lambda(z)^{n}<\varphi(z), f>v(z)+Q(z)^{n} f
$$

We can say much more on eigenvalues and eigenvectors when $z=\theta$ is real. At this point, we need to show that for every positive function $f \in \mathcal{B}$ with $f \neq 0$, there exists a positive linear form $\varphi \in \mathcal{B}^{\star}$ such that $\left.\langle\varphi, f\rangle\right\rangle 0$. In the context of [15], since functions are defined everywhere, there exists $x \in X$ such that $f(x)>0$, and so the Dirac mass $\delta_{x}$ does the job. In our context, Dirac masses are not available, but the reference measure is usable, since necessarily $\langle m, f>>0$, otherwise, $f$ would be $0 m$-ae, and so $f=0$ in $\mathcal{B}$. This was not the case in [15] because they consider functions defined everywhere, and not classes of equivalence. We can also use arguments from complex Banach lattice theory [22] : a modification of the Hahn-Banach theorem shows that there exists a positive bounded linear form $\varphi$ on $\mathcal{B}_{\mathbb{R}}=\{f \in \mathcal{B} / f(x) \in \mathbb{R} m$-ae $\}$, such that $\langle\varphi, f\rangle=1$, and then we can extend it on all $\mathcal{B}$. This argument could be employed in more abstract contexts, where the Banach space $\mathcal{B}$ consists of distributions-like objects and when we don't have a good knowledge of its topological dual.

Proposition 5. There exists $0<\theta_{1}<\theta_{0}$ such that for every $\theta \in \mathbb{R}$ with $|\theta|<\theta_{1}$, we have $\lambda(\theta)>0$. Furthermore, $v($.$) and \varphi($.$) can be redefined such that v(\theta) \geq 0$, $\varphi(\theta) \geq 0$.

Proof. As $P_{\theta}$ is a real operator, we have $P_{\theta} \bar{f}=\overline{P_{\theta} f}$ for all $f \in \mathcal{B}$. So, we have $P_{\theta} \overline{v(\theta)}=\overline{P_{\theta} v(\theta)}=\overline{\lambda(\theta)} \overline{v(\theta)}$. Since $\lambda(\theta)$ is the unique eigenvalue of $P_{\theta}$ with maximal modulus, we get $\overline{\lambda(\theta)}=\lambda(\theta)$, and hence $\lambda(\theta) \in \mathbb{R}$. Since $\lambda(0)=1$, by a continuity argument, we obtain $\lambda(\theta)>0$ for small $\theta$. For $z \in \mathbb{C}$ small enough, $\langle\varphi(z), \mathbb{1}>\neq 0$. We define $\tilde{v}(z)=<\varphi(z), \mathbb{1}>v(z)$ and $\tilde{\varphi}(z)=<\varphi(z), \mathbb{1}>^{-1} \varphi(z)$. Those new eigenfunctions satisfy obviously the conclusions of the previous proposition. We have just to prove that $\tilde{v}(\theta)$ and $\tilde{\varphi}(\theta)$ are positive for $\theta \in \mathbb{R}$ small enough. By the spectral decomposition of $P_{\theta}$, we see that $\lambda(\theta)^{-n} P_{\theta}^{n} \mathbb{1}$ goes to $\tilde{v}(\theta)$ in $\mathcal{B}$, and hence in $L^{1}(m)$. We then get $\tilde{v}(\theta) \geq 0$ because $P_{\theta}$ is a positive operator and $\lambda(\theta)$ is positive too. Now, 
let $\psi(\theta) \in \mathcal{B}^{\star}$ positive such that $\left\langle\psi(\theta), \tilde{v}(\theta)>=1\right.$. Then, $\lambda(\theta)^{-n}\left(P_{\theta}^{\star}\right)^{n} \psi(\theta)$ goes to $<\psi(\theta), v(\theta)>\varphi(\theta)=\tilde{\varphi}(\theta)$, which proves that $\tilde{\varphi}(\theta)$ is a positive linear form.

We denote

$$
\Lambda(\theta)=\log \lambda(\theta)
$$

We then have

Proposition 6. There exists $0<\theta_{2}<\theta_{1}$ such that for every $\theta \in \mathbb{B}$ with $|\theta|<\theta_{2}$ and every $f \in \mathcal{B}$ with $f \geq 0$ and $\int f d m=1$, we have

$$
\lim _{n \rightarrow \infty} \frac{1}{n} \log \int e^{\theta S_{n}} f d m=\Lambda(\theta)
$$

Proof. We have the identity

$$
\begin{aligned}
\int e^{\theta S_{n}} f d m=<m, P_{\theta}^{n}(f)> & =\lambda(\theta)^{n}<\varphi(\theta), f><m, v(\theta)>+<m, Q(\theta)^{n} f> \\
& =\lambda(\theta)^{n}\left(<\varphi(\theta), f><m, v(\theta)>+\lambda(\theta)^{-n}<m, Q(\theta)^{n} f>\right)
\end{aligned}
$$

All involved quantities are positive, hence we can write

$$
\frac{1}{n} \log \int e^{\theta S_{n}} f d m=\log \lambda(\theta)+\frac{1}{n} \log \left(<\varphi(\theta), f><m, v(\theta)>+\lambda(\theta)^{-n}<m, Q(\theta)^{n} f>\right)
$$

Since

$$
\lim _{\theta \rightarrow 0}<\varphi(\theta), f><m, v(\theta)>=1
$$

and since the spectral radius of $Q(\theta)$ is strictly less than $\lambda(\theta)$, it's easy to see that for $\theta$ small enough, we have

$$
\lim _{n \rightarrow \infty} \frac{1}{n} \log \left(<\varphi(\theta), f><m, v(\theta)>+\lambda(\theta)^{-n}<m, Q(\theta)^{n} f>\right)=0
$$

In order to apply Gartner-Ellis theorem, we just have to show that $\Lambda$ is differentiable function, strictly convex in a neighborhood of 0 . Since $\lambda$ is real-analytic, $\Lambda$ is too. Computations from perturbation theory show that $\lambda^{\prime}(0)=\int \phi d \mu=0$ and $\lambda^{\prime \prime}(0)=\sigma^{2}$, so we have $\Lambda^{\prime \prime}(0)=\frac{\lambda^{\prime \prime}(0) \lambda(0)-\lambda^{\prime}(0)^{2}}{\lambda(0)^{2}}=\sigma^{2}>0$ and we can now apply the following local version of Gartner-Ellis theorem, whose proof can be found in lemma XIII.2 in [15] :

\footnotetext{
${ }^{2}$ See corollaries III.11 and III.6 in [15].
} 
Proposition 7 . For all $n \geq 1$, denote by $\mathbb{P}_{n}$ a probability measure on some measurable space $(\Omega, \mathcal{T})$, by $\mathbb{E}_{n}$ the corresponding expectation operator and by $S_{n}$ a real valued random variable. Assume that on some interval $\left[-\theta_{\Lambda}, \theta_{\Lambda}\right], \theta_{\Lambda}>0$, we have

$$
\lim _{n \rightarrow \infty} \frac{1}{n} \log \mathbb{E}_{n}\left[\exp \left(\theta S_{n}\right)\right]=\Lambda(\theta),
$$

where $\Lambda$ is a strictly convex continuously differentiable function satisfying $\Lambda^{\prime}(0)=0$. Define $\epsilon_{+}=\frac{\Lambda\left(\theta_{\Lambda}\right)}{\theta_{\Lambda}}>0, \epsilon_{-}=\frac{\Lambda\left(\theta_{\Lambda}\right)}{\theta_{\Lambda}}<0$ and $c(\epsilon)=\sup _{|\theta| \leq \theta_{\Lambda}}\{\theta \epsilon-\Lambda(\theta)\}$. Then $c$ is a positive function, strictly convex on $\left[\epsilon_{-}, \epsilon_{+}\right]$, continuous, vanishing only at 0 , and, for all $0<\epsilon<\epsilon_{0}=\epsilon_{+}$, we have

$$
\lim _{n \rightarrow \infty} \frac{1}{n} \log \mathbb{P}_{n}\left(S_{n}>n \epsilon\right)=-c(\epsilon)
$$

We now prove our Theorem 2.

Central Limit Theorem. By Levy's continuity theorem, it suffices to show that for all $t \in \mathbb{R}$

$$
\lim _{n \rightarrow \infty} \int e^{i t \frac{S_{n}}{\sqrt{n}}} f d m=e^{-\frac{t^{2} \sigma^{2}}{2}}
$$

We have

$$
\int e^{i t \frac{S_{n}}{\sqrt{n}}} f d m=<m, P_{\frac{i t}{\sqrt{n}}}^{n}(f)>=\lambda\left(\frac{i t}{\sqrt{n}}\right)^{n}<\varphi\left(\frac{i t}{\sqrt{n}}\right), f><m, v\left(\frac{i t}{\sqrt{n}}\right)>+<m, Q\left(\frac{i t}{\sqrt{n}}\right)^{n} f>
$$

We just have to prove that

$$
\lim _{n \rightarrow \infty} \lambda\left(\frac{i t}{\sqrt{n}}\right)^{n}=e^{-\frac{t^{2} \sigma^{2}}{2}}
$$

But the Taylor's expansion says that in a complex neighborhood of 0

$$
\lambda(z)=\lambda(0)+\lambda^{\prime}(0) z+\frac{\lambda^{\prime \prime}(0)}{2} z^{2}+z^{2} \eta(z)=1+\frac{\sigma^{2} z^{2}}{2}+z^{2} \eta(z)
$$

where $\lim _{z \rightarrow 0} \eta(z)=0$. Then, a standard computation concludes the proof.

\section{Application to Uniformly expanding maps}

The main application of our Theorem 1 will be to multidimensional piecewise uniformly expanding maps, in particular when we equip them with the space of the quasi-Hölder functions. This space, introduced by Keller [17], developed by Blank [3] and successfully applied by Saussol [25] and successively by Buzzi [7] (see also [8]) and Tsujii [28], reveals to be very useful to control the oscillations of a function under the iteration of the transfer operator across the discontinuities of the map. Moreover it verifies the algebraic assumption 3 in Section 2 above; it is not straightforward to replace this condition and in order to fit with the Hennion-Hervé theory, if one 
uses the more conventional spaces of bounded variation functions (see for instance [1, 5, 9, 21, 23]) or the Sobolev spaces [27], and this topic deserves to be investigated in the future 3 .

Let us now recall the precise definitions of our system by following closely the assumptions imposed in [25]. Let $M \subset \mathbb{R}^{d}$ be a compact subset with $\overline{\operatorname{int} M}=M$ and piecewise $C^{1}$ boundary. We denote by $d$ the Euclidean distance and by $m$ the Lebesgue measure on $\mathbb{R}^{d}$. We can assume without loss of generality that $m(M)=1$. For $A \subset M$ and $\epsilon>0$, we denote $B_{\epsilon}(A)=\left\{x \in \mathbb{R}^{d} / d(x, A) \leq \epsilon\right\}$. Let $T: M \rightarrow M$ a measurable application, and suppose there exists $0<\alpha \leq 1$ such that for some small enough $\epsilon_{0}$ we have :

(1) There are finitely many disjoint open sets $U_{i} \subset M$ with $m\left(M \backslash \cup_{i} U_{i}\right)=0$ such that for each $i, T_{i}:=\left.T\right|_{U_{i}} \rightarrow M$ is $C^{1+\alpha}$ and can be extended on a neighborhood $V_{i}$ of $U_{i}$ to a $C^{1+\alpha}$ map $T_{i}: V_{i} \rightarrow \mathbb{R}^{d}$ such that $B_{\epsilon_{0}}\left(T_{i} U_{i}\right) \subset$ $T_{i}\left(V_{i}\right)$. Moreover, each $T_{i}: V_{i} \rightarrow \mathbb{R}^{d}$ is injective with $C^{1+\alpha}$ inverse;

(2) There exists $c>0$ such that for any $i$, and any $x, y \in T\left(U_{i}\right)$ with $d(x, y) \leq \epsilon_{0}$ we have

$$
\left|\operatorname{det} D T_{i}^{-1}(x)-\operatorname{det} D T_{i}^{-1}(y)\right| \leq c\left|\operatorname{det} D T_{i}^{-1}(x)\right| d(x, y)^{\alpha} ;
$$

(3) There exists $s(T)<1$ such that

$$
\sup _{i} \sup _{x \in T_{i}\left(V_{i}\right)}\left\|D T_{i}^{-1}(x)\right\|<s(T)
$$

(4) Boundaries of $U_{i}$ are piecewise $C^{1}$ codimension one embedded compact submanifolds and we have $\eta_{0}(T)<1$ where

$$
\begin{gathered}
\eta_{0}(T)=s(T)^{\alpha}+\frac{4 s(T)}{1-s(T)} Y(T) \frac{\gamma_{d-1}}{\gamma_{d}} \\
Y(T)=\sup _{x \in \mathbb{R}^{d}} \sum_{i} \sharp\left\{\text { smooth pieces intersecting } \partial U_{i} \text { and containing } x\right\} \\
\text { and } \gamma_{d}=\frac{\pi^{d / 2}}{(d / 2) !} \text { is the } d \text {-volume of the } d \text {-dimensional unit ball of } \mathbb{R}^{d} \text {. }
\end{gathered}
$$

The last condition can be greatly weakened, but the condition in [25] is of a very abstract nature, and it's more easy to handle with this one when the boundaries of the $U_{i}$ are smooth. We define then the functional space on which acts the transfer

\footnotetext{
${ }^{3}$ In fact, if we check the proof, we only need the fact that $\mathcal{B}$ is Banach algebra and $\phi \in \mathcal{B}$ to prove that the operators $P_{z}$ are well defined and holomorphic in $z$. So we can suppose the weaker assumption that $\phi$ is such that $P_{z}$ define a holomorphic family of bounded operators on $\mathcal{B}$ for $z$ in a complex neighbourhood of 0 .
} 
operator. Let $f \in L^{1}\left(\mathbb{R}^{d}\right)$. If $A \subset \mathbb{R}^{d}$ is a Borel subset, we define the oscillation of $f$ over $A$ by

$$
\operatorname{osc}(f, A)=\operatorname{ess}_{x_{1}, x_{2} \in A}\left|f\left(x_{1}\right)-f\left(x_{2}\right)\right|
$$

where the essential supremum is taken with respect to the product measure $m \times m$ on $A \times A$. We get a lower semi-continuous and hence measurable function $x \rightarrow$ $\operatorname{osc}\left(f, B_{\epsilon}(x)\right)$. We set

$$
|f|_{\alpha}=\sup _{0<\epsilon \leq \epsilon_{0}} \frac{1}{\epsilon^{\alpha}} \int_{\mathbb{R}^{d}} \operatorname{osc}\left(f, B_{\epsilon}(x)\right) d x
$$

We define

$$
V_{\alpha}\left(\mathbb{R}^{d}\right)=\left\{f \in L^{1}\left(\mathbb{R}^{d}\right) /|f|_{\alpha}<\infty\right\}
$$

and

$$
V_{\alpha}(M)=\left\{f \in V_{\alpha}\left(\mathbb{R}^{d}\right) / \operatorname{supp} f \subset M\right\}
$$

both endowed with the norm $\|f\|_{\alpha}=\|f\|_{L_{m}^{1}}+|f|_{\alpha}$. Adapting proofs from [17], we can show that $V_{\alpha}(M)$ is Banach space, with compact injection in $L^{1}(M)$. It's proven in [25] that $V_{\alpha}(M)$ is also a Banach algebra, and it's obviously a Banach lattice. So, if we want to apply our previous results to those maps, we are left to prove that the transfer operator for $T$ acts on $V_{\alpha}(M)$ and is quasi-compact of diagonal type. But Saussol proved in this context a Lasota-Yorke inequality (lemma 4.1 in [25]) which implies the quasi-compactness of the Perron-Frobenius operator. Hence, assuming that the system is mixing, we get the central limit theorem and the large deviations principle for bounded real observables in $V_{\alpha}(M)$.

\section{REFERENCES}

[1] K. Adl-Zarabi, Absolutely continuous invariant measures for piecewise expanding $C^{2}$ transformations in $\mathbb{R}^{n}$ on domains with cusps on the boundaries, Ergodic Theory and Dynamical Systems, 16, (1996), 1-18

[2] J.F. Alves, J. Freitas, S. Luzzatto, S. Vaienti, From rates of mixing to recurrence times via large deviations, Adv. in Math., 228, (2011), 1203-1236

[3] M. Blank, Stochastic properties of deterministic dynamical systems, Sov. Sci. Rev. C Maths/Phys., 6, (1987), 243-271

[4] A. Boyarsky, P. Góra, Laws of Chaos : Invariant Measures and Dynamical Systems in One Dimension, Probability and its Applications, Birkhauser, 1997

[5] A. Boyarsky and P. Gora, Absolutely continuous invariant measures for piecewise expanding $C^{2}$ transformations in $\mathbb{R}^{n}$, Israel J. Math., 67, (1987), 272-286

[6] A. Broise, Transformations dilatantes de l'intervalle et théorèmes limites, Astérisque, 238, (1996), 5-110.

[7] J. Buzzi, Absolutely continuous invariant probability measures for arbitrary expanding piecewise $\mathbf{R}$-analytic mappings of the plane, Ergodic Theory and Dynamical Systems, 20, (2000), 697-708

[8] J. Buzzi and G. Keller, Zeta functions and transfer operators for multidimensional piecewise affine and expanding maps, Ergodic Theory and Dynamical Systems, 21, (2001), 689-716 
[9] W.J. Cowieson, Stochastic stability for piecewise expanding maps in $\mathbb{R}^{d}$, Nonlinearity, 13.,(2000), 1745-1760

[10] A. Dembo, O. Zeitouni, Large Deviations, Techniques and Applications, Applications of Mathematics 38, Springer-Verlag, 2nd Edition, 1998

[11] N. Dunford, J.T. Schwartz, Linear Operators, Part I : General Theory, Wiley, 1957

[12] R.S. Ellis, Entropy, Large Deviations and Statistical Mechanics, Springer-Verlag, New-York, 1985

[13] M.I. Gordin, The central limit theorem for stationary processes, Soviet. Math. Dokl., 10, 11741176

[14] H. Hennion, Sur un théorème spectral et son application aux noyaux Lipschitziens, Proceedings of the A.M.S., 118 (1993), 627-634

[15] H. Hennion, L. Hervé, Limit theorems for Markov chains and Stochastic Properties of Dynamical Systems by Quasicompactness, Lect. Notes Math., 1766, (2001), Springer-Verlag

[16] C.T. Ionescu-Tulcea, G. Marinescu, Théorie ergodique pour des classes d'opérations non complètement continues, Ann. Math 52 (1950), 140-147

[17] G. Keller, Generalized bounded variation and applications to piecewise monotonic transformations, Z. Wahr. verw. Geb., 69, (1985), 461-478

[18] A. Lasota, M.C. Mackey, Chaos, Fractals and Noise: Stochastic Aspects of Dynamics, SpringerVerlag, 1994

[19] A. Lasota, J.-A. Yorke, On the existence of invariant measures for piecewise monotonic transformations, Trans. Amer. Math. Soc., 186, (1973), 481-488

[20] C. Liverani, Central Limit Theorem for Deterministic Systems, Pitman Research Notes in Mathematics Series, 362, (1996), 56-75

[21] C. Liverani, Multidimensional expanding maps with singularities: a pedestrian approach, to appear in Ergodic Theory and Dynamical Systems

[22] P. Meyer-Nieberg, Banach Lattices, Universitext, Springer-Verlag, Berlin, 1991

[23] H. Poppe, P. Gora and A. Boyarsky, Inadequacy of the bounded variation technique in the ergodic theory of higher-dimensional transformations in $\mathbb{R}^{n}$, Israel J. Math., 3, (1990), 10811087

[24] L. Rey-Bellet, L.S. Young, Large deviations in non-uniformly hyperbolic dynamical systems, Ergodic Theory and Dynamical Systems 28 (2008), 587-612

[25] B. Saussol, Absolutely continuous invariant measures for multidimensional expanding maps, Israel J. Math., 116, (2000), 223-248

[26] M. Thaler, Estimates of the invariant densities of endomorphisms with indifferent periodic points, Israel J. Math., 37, (1980), 303-314

[27] D. Thomine, A spectral gap for transfer operators of piecewise expanding maps, Discrete and Continuous Dynamical Systems (A), 30, (2011), 917-944

[28] M. Tsujii, Absolutely continuous invariant measures for expanding piecewise linear maps, Invent. Math., 143, (2001), 349-373

[29] L.S. Young, Statistical properties of dynamical systems with some hyperbolicity, Ann. of Math. (2) 147 (1998) 585-650. 
R. Aimino, UmR-6207 Centre de Physique Théorique, CNRS, Universités D’AixMarseille, Université du Sud, Toulon-VAr and FRUMAM, FÉdéderation de Recherche Des Unités de Mathématiques de Marseille, CPT, Luminy Case 907, F-13288 Marseille Cedex 9, France

E-mail address: romain.aimino@orange.fr

S. Vaienti, UMR-6207 Centre de Physique Théorique, CNRS, Universités d’AixMarseille, Université du Sud, Toulon-Var and FRUMAM, FÉdéderation de Recherche des Unités de Mathématiques de Marseille, CPT, Luminy Case 907, F-13288 Marseille Cedex 9, France

E-mail address: vaienti@cpt.univ-mrs.fr 